\title{
Directed immersions of closed manifolds
}

\author{
MOHAMMAD GHOMI
}

\begin{abstract}
Given any finite subset $X$ of the sphere $\mathbf{S}^{n}, n \geq 2$, which includes no pairs of antipodal points, we explicitly construct smoothly immersed closed orientable hypersurfaces in Euclidean space $\mathbf{R}^{n+1}$ whose Gauss map misses $X$. In particular, this answers a question of M Gromov.
\end{abstract}

53A07, 53C42; 57R42, 58K15

\section{Introduction}

To every $\left(\mathcal{C}^{1}\right)$ immersion $f: M^{n} \rightarrow \mathbf{R}^{n+1}$ of a closed oriented $n$-manifold $M$, there corresponds a unit normal vector field or Gauss map $G_{f}: M \rightarrow \mathbf{S}^{n}$, which generates a set $G_{f}(M) \subset \mathbf{S}^{n}$ known as the spherical image of $f$. Conversely, one may ask (see Gromov [8, page 3]): for which sets $A \subset \mathbf{S}^{n}$ is there an immersion $f: M \rightarrow \mathbf{R}^{n+1}$ such that $G_{f}(M) \subset A$ ? Such a mapping would be called an $A$-directed immersion of $M$; see Eliashberg and Mishachev [1], Gromov [7], Rourke and Sanderson [13] and Spring [14]. It is well-known that when $A \neq \mathbf{S}^{n}, f$ must have double points (Section 4.1), and $M$ must be parallelizable, eg, $M$ can only be the torus $\mathbf{T}^{2}$ when $n=2$ (Section 4.2). Furthermore, the only known necessary condition on $A$ is the elementary observation that $A \cup-A=\mathbf{S}^{2}$, while there is also a sufficient condition due to Gromov [7, Theorem $\left(D^{\prime}\right)$, page 186]:

Condition 1.1 $A \subset \mathbf{S}^{n}$ is open, and there is a point $p \in \mathbf{S}^{n}$ such that the intersection of $A$ with each great circle passing through $p$ includes a (closed) semicircle.

A great circle is the intersection of $\mathbf{S}^{n}$ with a 2-dimensional subspace of $\mathbf{R}^{n+1}$. Note that, when $n \geq 2$, examples of sets $A \subset \mathbf{S}^{n}$ satisfying the above condition include those which are the complement of a finite set of points without antipodal pairs. Thus the spherical image of a closed hypersurface can be remarkably flexible. Like most h-principle or convex integration type arguments, however, the proof does not yield specific examples. It is therefore natural to ask, for instance:

Question 1.2 [7, page 186] Is there a "simple" immersion $\mathbf{T}^{2} \rightarrow \mathbf{R}^{3}$ whose spherical image misses the four vertices of a regular tetrahedron in $\mathbf{S}^{2}$ ? 
Here we give an affirmative answer to this question (Section 2), and more generally present a short constructive proof of the sufficiency of a slightly stronger version of Condition 1.1 for the existence of $A$-directed immersions of parallelizable manifolds $M^{n-1} \times \mathbf{S}^{1}$, where $M^{n-1}$ is closed and orientable. Any such manifold admits an immersion $f: M^{n-1} \rightarrow \mathbf{R}^{n} \times\{0\} \subset \mathbf{R}^{n+1}$ (Section 4.3). We then extend $f$ to $M^{n-1} \times \mathbf{S}^{1}$ by using the figure eight curve

$$
E_{\delta}(t):=(\cos (t), \delta \sin (2 t))
$$

to put a copy of $\mathbf{S}^{1} \simeq \mathbf{R} / 2 \pi$ in each normal plane of $f$, as described below. Note that the midpoint of $G_{E_{\delta}}\left(\mathbf{S}^{1}\right)$ is assumed to be at $(1,0)$; see Figure 1 which shows $E_{1 / 2}$ and its spherical image. Further, the unit normal bundle of $f$ may be naturally identified with the pencil of great circles of $\mathbf{S}^{n}$ passing through $(0, \ldots, 0,1)$.
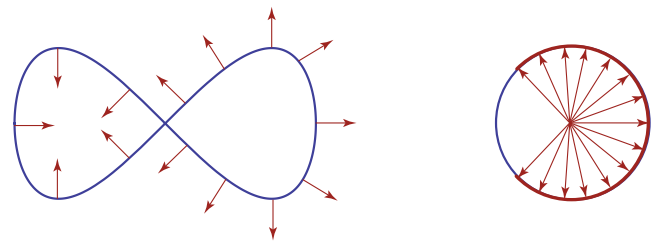

Figure 1

Theorem 1.3 Let $A \subset \mathbf{S}^{n}$ satisfy Condition 1.1 with respect to $p=(0, \ldots, 0,1)$. Further, if $n \geq 3$, suppose that the semicircle in Condition 1.1 contains $p$, or that no great circle through $p$ is contained in $A$. Let $f: M^{n-1} \rightarrow \mathbf{R}^{n} \times\{0\} \subset \mathbf{R}^{n+1}$ be a smooth $\left(\mathcal{C}^{\infty}\right)$ immersion of a closed orientable $(n-1)$-manifold, and, for every $q \in M$, let $C_{q} \subset \mathbf{S}^{n}$ be the unit normal space of $f$ at $q$. Then there is a smooth orthogonal frame $\left\{N_{i}: M \rightarrow \mathbf{S}^{n}\right\}, i=1,2$, for the normal bundle of $f$ such that the semicircle in $C_{q}$ centered at $N_{1}(q)$ lies in $A$. For any such frame, and sufficiently small $\varepsilon, \delta>0$,

$$
F(q, t):=f(q)+\varepsilon \sum_{i=1}^{2} E_{\delta}^{i}(t) N_{i}(q)
$$

yields a smooth $A$-directed immersion $M \times \mathbf{S}^{1} \rightarrow \mathbf{R}^{n+1}$, where $E_{\delta}^{i}$ are the components of the figure eight curve $E_{\delta}$ given by (1).

It is not known if Condition 1.1 is necessary for the existence of $A$-directed closed hypersurfaces, and the question posed in the first paragraph is open, even for $n=2$. See Ghomi [3; 4] and Ghomi and Tabachnikov [6] for some other recent results on Gauss maps of closed submanifolds, Ghomi [2], Hartman and Nirenberg [9], Milnor [11] and $\mathrm{Wu}$ [16] for still more studies of spherical images, and Spring [15] for historical background. 


\section{Example}

If $A=\mathbf{S}^{2} \backslash X$ for a finite set $X$ without antipodal pairs, we may always find a point $p \in \mathbf{S}^{2}$ with respect to which $A$ satisfies the hypothesis of Theorem 1.3 (eg, let $p \notin X$ be in the complement of all great circles which pass through at least two points of $X$ other than $-p$ ). After a rigid motion (which may be arbitrarily small) we may assume that $p=(0,0,1)$ or $(0,0,-1)$, and let $f(\theta):=(\cos (\theta), \sin (\theta), 0)$ be the standard immersion of $\mathbf{S}^{1} \simeq \mathbf{R} / 2 \pi$ in $\mathbf{R}^{3}$. Then the desired framing for the normal bundle of $f$ may always take the form

$$
N_{1}(\theta):=f^{\prime}(\theta) \times N_{2}(\theta), \quad N_{2}(\theta):=\frac{(\cos (\theta), \sin (\theta), z(\theta))}{\sqrt{1+z^{2}(\theta)}},
$$

where $z: \mathbf{R} / 2 \pi \rightarrow \mathbf{R}$ is a smooth function with $z(\theta)=-z(\theta+\pi)$ and such that $X$ is contained entirely in one of the components of $\mathbf{S}^{2}-N_{2}\left(\mathbf{S}^{1}\right)$. For instance, when $X$ is the vertices of a regular tetrahedron, we may set $z(\theta):=\cos (3 \theta)$ in (3). Then, for $\varepsilon, \delta \leq$ $1 / 8$, the mapping $F(\theta, t)$ given by (2) yields an immersion $\mathbf{T}^{2} \simeq \mathbf{R} / 2 \pi \times \mathbf{R} / 2 \pi \rightarrow \mathbf{R}^{3}$ which answers Question 1.2. The resulting surface, for $\varepsilon=\delta=1 / 8$, is depicted in Figure 2 together with its spherical image (note that here $p=(0,0,-1)$ ). To find $z(\theta)$
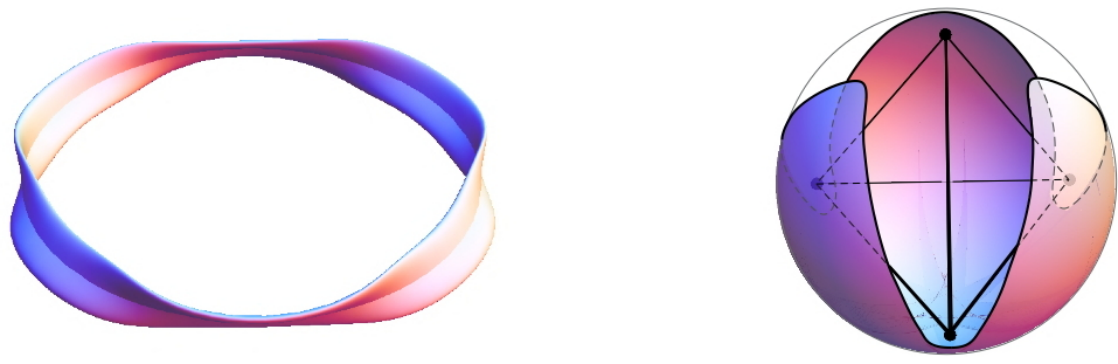

Figure 2

in general, we may order the points in $X^{\prime} \cup-X^{\prime}$, where $X^{\prime}:=X \backslash\{-p\}$, according to their "longitude" $\theta$, and connect them by geodesic segments to obtain a simple closed symmetric curve $\gamma(\theta)$. A perturbation of $\gamma$ then yields a smooth symmetric curve $\tilde{\gamma}$ such that $X$ is contained in one of the components of $\mathbf{S}^{2}-\tilde{\gamma}\left(\mathbf{S}^{1}\right)$. The third coordinate of $\tilde{\gamma}$ gives our desired height function $z$.

\section{Proof of Theorem 1.3}

3.1 First we construct the frame $\left\{N_{i}\right\}$. For every $q \in M, C_{q}$ is a great circle passing through $p$. So it contains a semicircle in $A$ by assumption (Condition 1.1). Let 
$m_{q} \subset C_{q}$ be the set of midpoints of all such semicircles. We need to find a smooth map $N_{1}: M \rightarrow \mathbf{S}^{n}$ such that $N_{1}(q) \in m_{q}$ for all $q \in M$. To this end note that $m_{q}$ is open and connected. Further, if $m_{q}$ contains any pairs of antipodal points, then $m_{q}=C_{q}$; otherwise, $m_{q}$ lies in the interior a semicircle of $C_{q}$. Consequently,

$$
\operatorname{Cone}\left(m_{q}\right):=\left\{\lambda x \mid x \in m_{q} \text { and } \lambda \geq 0\right\},
$$

is a convex set in $\mathbf{R}^{n+1}$. In particular, for any finite set of points $x_{i} \in \operatorname{Cone}\left(m_{q}\right)$ and numbers $\lambda_{i} \geq 0, \sum_{i} \lambda_{i} x_{i} \in \operatorname{Cone}\left(m_{q}\right)$. Now let $B$ be the set of all points $q \in M$ such that $m_{q} \neq C_{q}$. Then $B$ is closed (and therefore compact) since $M \backslash B$ is open; indeed the set of great circles contained in $A$ is open, since $A$ is open. Further note that for any point $q \in M$, normal vector $x \in m_{q}$, and continuous local extension $v$ of $x$ to a normal vector field of $M$, we have $v\left(q^{\prime}\right) \in m_{q^{\prime}}$ for all $q^{\prime}$ in an open neighborhood $U$ of $q$ (because the set of semicircles contained in $A$ is open). Let $\left\{v_{i}: U_{i} \rightarrow \mathbf{S}^{n}\right\}$, $i=1, \ldots, k$, be a finite collection of such local vector fields so that $\bigcup_{i} U_{i}$ covers $B$ and $v_{i}$ are smooth. Also let $\left\{\phi_{i}: M \rightarrow \mathbf{R}\right\}$ be a smooth partition of unity subordinate to $\left\{U_{i}\right\}$, and, for $q \in \bigcup_{i} U_{i}$, set

$$
N_{1}(q):=\frac{\sum_{i=1}^{k} \phi_{i}(q) v_{i}(q)}{\left\|\sum_{i=1}^{k} \phi_{i}(q) v_{i}(q)\right\|} .
$$

If $q \in B$, then $v_{i}(q) \in m_{q}$ which lies in the interior of a semicircle $S \subset C_{q}$, and so $\left\|\sum_{i=1}^{k} \phi_{i}(q) v_{i}(q)\right\| \neq 0$. Indeed, if $x$ is the midpoint of $S$, then

$$
\left\langle\sum_{i=1}^{k} \phi_{i}(q) v_{i}(q), x\right\rangle=\sum_{i=1}^{k} \phi_{i}(q)\left\langle v_{i}(q), x\right\rangle>0 .
$$

Thus $N_{1}$ is well defined (and smooth) on an open neighborhood $V$ of $B$. Further, $N_{1}(q) \in m_{q}$, for all $q \in V$, since Cone $\left(m_{q}\right)$ is convex. In particular we are done if $B=M$; otherwise, note that we may write

$$
N_{1}(q)=\cos (\theta(q)) p+\sin (\theta(q)) G_{f}(q),
$$

for some function $\theta: V \rightarrow \mathbf{R}$, since $G_{f}$ is well defined due to the orientability of $M$, and thus $\left\{p, G_{f}(q)\right\}$ forms an orthonormal basis for the normal plane $d f\left(T_{q} M\right)^{\perp}$. Further, it is easy to see that we may choose $\theta$ continuously (and therefore smoothly) if $n=2$. This also holds for $n>2$ if each $C_{q}$ contains a semicircle passing through $p$; for then $\theta$ is uniquely determined within the range $[-\pi / 2, \pi / 2]$. Indeed, we may choose the vectors $v_{i}$ above so that $\left\langle v_{i}(q), p\right\rangle \geq 0$ which would in turn yield that $\left\langle N_{1}(q), p\right\rangle \geq 0$. Now let $V^{\prime}$ be an open neighborhood of $B$ with closure $\overline{V^{\prime}} \subset V$. Using Tietze's theorem, followed by a perturbation and a gluing, we may extend $\left.\theta\right|_{V^{\prime}}$ smoothly to all of $M$. Then (4) yields the desired vector field on $M$, since for any 
$q \in M \backslash B, N_{1}(q) \in C_{q}=m_{q}$. Finally, set

$$
N_{2}(q):=\sin (\theta(q)) p-\cos (\theta(q)) G_{f}(q) .
$$

3.2 It remains to show that $G_{F}\left(M \times \mathbf{S}^{1}\right) \subset A$, for small $\varepsilon, \delta>0$. For all $q \in M, C_{q} \cap A$ contains an arc of length $\geq \pi+\alpha$ with midpoint $N_{1}(q)$ for some uniform constant $\alpha>0$. Indeed, if we let $g(q)$ be the supremum of lengths of all $\operatorname{arcs}$ in $C_{q} \cap A$ with midpoint $N_{1}(q)$, then $g: M \rightarrow \mathbf{R}$ is lower semicontinuous, ie, $\lim _{q \rightarrow q_{0}} g(q) \geq g\left(q_{0}\right)$, since $A$ is open. Thus, since $g>\pi$ and $M$ is compact, $g \geq \pi+\alpha$. Now choose $\delta>0$ so small that the length $\ell$ of the spherical image of $E_{\delta}$ is $\leq \pi+\alpha$ (this is possible since $\ell \rightarrow \pi$ as $\delta \rightarrow 0)$. Next, for $(q, t) \in M \times \mathbf{S}^{1}$, let $\widetilde{G}_{F}(q, t)$ be the normalized projection of $G_{F}(q, t)$ into $d f\left(T_{q} M\right)^{\perp}$, ie,

$$
\tilde{G}_{F}(q, t):=\frac{\sum_{i=1}^{2}\left\langle G_{F}(q, t), N_{i}(q)\right\rangle N_{i}(q)}{\sqrt{\sum_{i=1}^{2}\left\langle G_{F}(q, t), N_{i}(q)\right\rangle^{2}}} .
$$

Also, for fixed $t \in \mathbf{S}^{1}$, let $F_{t}(q):=F(q, t)$. Then, by the tubular neighborhood theorem, $F_{t}: M \rightarrow \mathbf{R}^{n+1}$ is a smooth immersion for small $\varepsilon$. Further, as $\varepsilon \rightarrow 0, F_{t}$ converges to $f$ with respect to the $\mathcal{C}^{1}$-topology. Thus, for each $q \in M$, the normal plane $d F_{t}\left(T_{q} M\right)^{\perp}$ (which contains $\left.G_{F}(q, t)\right)$ converges to $d f\left(T_{q} M\right)^{\perp}$. Consequently $G_{F}$ is well-defined for small $\varepsilon$, and converges to $\widetilde{G}_{F}$ as $\varepsilon \rightarrow 0$. So it suffices to check that $\widetilde{G}_{F}\left(M \times \mathbf{S}^{1}\right) \subset A$, which follows from our choice of $\delta$. Indeed for each fixed $q \in M$, $\widetilde{G}_{F}\left(\{q\} \times \mathbf{S}^{1}\right)$ is the spherical image of the figure eight curve $\sum_{i=1}^{2} E_{\delta}^{i}(t) N_{i}(q)$ in $d f\left(T_{q} M\right)^{\perp}$, which is an arc of $C_{q}$ with midpoint $N_{1}(q)$ and length $\leq \pi+\alpha$.

\section{Notes}

4.1 It is well-known that $G_{f}(M)=\mathbf{S}^{n}$ for any embedding $f: M^{n} \rightarrow \mathbf{R}^{n+1}$ of a closed oriented $n$-manifold [7, page 187]. More generally, this also holds for "Alexandrov embeddings", ie, immersions $f: M \rightarrow \mathbf{R}^{n+1}$ which may be extended to an immersion $\bar{f}: \bar{M} \rightarrow \mathbf{R}^{n+1}$ of a compact $(n+1)$-manifold $\bar{M}$ with $\partial \bar{M}=M$. Indeed if $v$ is any vector field along $M$ which points "outward" with respect to $\bar{M}$, then for $p \in M$, the normalized projection of $d f(v(p))$ into the line $d f\left(T_{p} M\right)^{\perp}$ defines a normal vector field $M \rightarrow \mathbf{S}^{n}$ which coincides with $G_{f}$ (after a reflection of $G_{f}$ if necessary). Then, for any $u \in \mathbf{S}^{n}$, if $p$ is a point which maximizes the height function $\langle\cdot, u\rangle$ on $M$, we have $G_{f}(p)=u$. On the other hand, being only regularly homotopic to an embedding, is not enough to ensure that $G_{f}(M)=\mathbf{S}^{n}$. Indeed the example in Figure 2 is regularly homotopic to an embedded torus of revolution by Pinkall [12]. 
4.2 If $G_{f}(M) \neq \mathbf{S}^{n}$ for an immersion $f: M^{n} \rightarrow \mathbf{R}^{n+1}$ of an oriented $n$-manifold, then, as is well-known (see Milnor [11]), $M$ must be parallelizable. Here we include a brief geometric argument for this fact. If $(0, \ldots, 0,1) \notin G_{f}(M)$, we may define a continuous map $F: T M \rightarrow \mathbf{R}^{n} \simeq \mathbf{R}^{n} \times\{0\} \subset \mathbf{R}^{n+1}$ as follows; cf [5, Lemma 2.2]. There is a continuous map $\rho: \mathbf{S}^{n} \backslash\{(0, \ldots, 0,1)\} \rightarrow \mathrm{SO}(n+1), u \mapsto \rho_{u}$ such that $\rho_{u}(u)=(0, \ldots, 0,-1)$. Let $\pi: T M \rightarrow M$ be the canonical projection, and for $X \in T M$ set $F(X):=\rho_{G_{f}(\pi(X))}(d f(X))$. Also let $F_{p}:=\left.F\right|_{T_{p} M}$. Then $\left\{F_{p}^{-1}\left(e_{i}\right)\right\}$, where $\left\{e_{i}\right\}$ is a fixed basis of $\mathbf{R}^{n}$, gives a framing for $T M$ as desired. So in particular, when $M$ is closed and $n=2$, we have $M=\mathbf{T}^{2}$. The last observation also follows from Gauss-Bonnet theorem via degree theory when $f$ is $\mathcal{C}^{2}$; since if $G_{f}(M) \neq \mathbf{S}^{2}$, then

$$
0=\operatorname{deg}\left(G_{f}\right)=\frac{1}{4 \pi} \int_{M} \operatorname{det}\left(d G_{f}\right)=\frac{1}{4 \pi} \int_{M} K=\frac{1}{2} \chi(M),
$$

where $K$ is the Gaussian curvature and $\chi$ is the Euler characteristic.

4.3 To generate some concrete examples of the immersions $f: M^{n-1} \rightarrow \mathbf{R}^{n} \simeq \mathbf{R}^{n} \times\{0\}$ in Theorem 1.3, note that if $f_{0}: M_{0}^{n-k-1} \rightarrow \mathbf{R}^{n-k} \times\{0\}$ is any immersion such that $f_{0}\left(M_{0}\right)$ is disjoint from the subspace $L:=\mathbf{R}^{n-k-1} \times\{(0,0)\}$, then spinning $f_{0}$ about $L$ yields an immersion $f_{1}: M_{0} \times \mathbf{S}^{1} \rightarrow \mathbf{R}^{n-k+1}$ given by

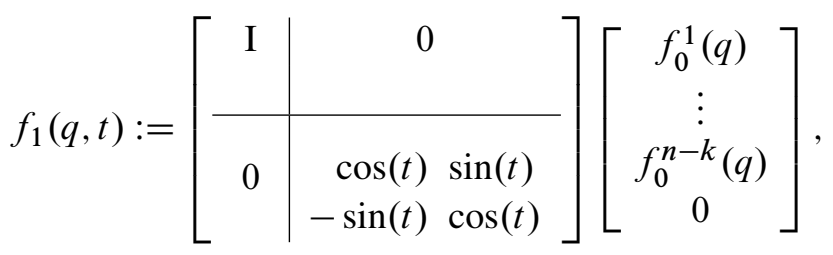

where $f_{0}^{i}$ are the components of $f_{0}$. Thus, for instance, one may inductively construct immersions of $\mathbf{S}^{n-k-1} \times \mathbf{T}^{k}$ in $\mathbf{R}^{n}$, for $k=1, \ldots, n-1$. More generally, if $M^{n-1} \times \mathbf{S}^{1}$ is parallelizable, then so is the open manifold $M^{n-1} \times(0,1)$, which may be immersed in $\mathbf{R}^{n}$ [10] by the h-principle [7], or more specifically, the "holonomic approximation theorem" of Eliashberg and Mishachev [1;5].

Acknowledgements The author thanks Misha Gromov for his interesting question in [7, page 186], and David Spring who first called the author's attention to that problem and pointed out a correction in an earlier draft of this work.

The research of the author was supported in part by NSF grant DMS-0806305.

\section{References}

[1] Y Eliashberg, N Mishachev, Introduction to the h-principle, Graduate Studies in Math. 48, Amer. Math. Soc. (2002) MR1909245 
[2] M Ghomi, Gauss map, topology, and convexity of hypersurfaces with nonvanishing curvature, Topology 41 (2002) 107-117 MR1871243

[3] M Ghomi, Shadows and convexity of surfaces, Ann. of Math. (2) 155 (2002) 281-293 MR1888801

[4] M Ghomi, Tangent bundle embeddings of manifolds in Euclidean space, Comment. Math. Helv. 81 (2006) 259-270 MR2208806

[5] M Ghomi, M Kossowski, $h$-principles for hypersurfaces with prescribed principal curvatures and directions, Trans. Amer. Math. Soc. 358 (2006) 4379-4393 MR2231382

[6] M Ghomi, S Tabachnikov, Totally skew embeddings of manifolds, Math. Z. 258 (2008) 499-512 MR2369041

[7] M Gromov, Partial differential relations, Ergebnisse der Math. und ihrer Grenzgebiete (3) 9, Springer, Berlin (1986) MR864505

[8] M Gromov, Spaces and questions, from: "Visions in mathematics: GAFA 2000 (Tel Aviv, 1999)”, (N Alon, J Bourgain, A Connes, M Gromov, V D Milman, editors), Geom. Funct. Anal., Special Volume, Part I (2000) 118-161 MR1826251

[9] P Hartman, L Nirenberg, On spherical image maps whose Jacobians do not change sign, Amer. J. Math. 81 (1959) 901-920 MR0126812

[10] M W Hirsch, On imbedding differentiable manifolds in euclidean space, Ann. of Math. (2) 73 (1961) 566-571 MR0124915

[11] J Milnor, On the immersion of $n$-manifolds in $(n+1)$-space, Comment. Math. Helv. 30 (1956) 275-284 MR0079268

[12] U Pinkall, Regular homotopy classes of immersed surfaces, Topology 24 (1985) 421434 MR816523

[13] C Rourke, B Sanderson, The compression theorem. II. Directed embeddings, Geom. Topol. 5 (2001) 431-440 MR1833750

[14] D Spring, Directed embeddings of closed manifolds, Commun. Contemp. Math. 7 (2005) 707-725 MR2175094

[15] D Spring, The golden age of immersion theory in topology: 1959-1973. A mathematical survey from a historical perspective, Bull. Amer. Math. Soc. (N.S.) 42 (2005) 163-180 MR2133309

[16] H Wu, The spherical images of convex hypersurfaces, J. Differential Geometry 9 (1974) 279-290 MR0348685

School of Mathematics, Georgia Institute of Technology

Atlanta GA 30332, USA

ghomi@math.gatech.edu

www. math.gatech.edu/ ghomi

Proposed: Yasha Eliashberg

Seconded: Leonid Polterovich, Dmitri Burago

Received: 25 October 2010 Accepted: 13 March 2011 